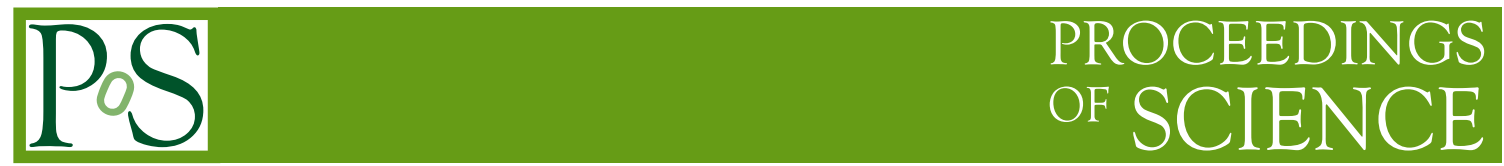

\title{
Chiral Polarization Scale at Finite Temperature
}

\section{Andrei Alexandru*}

The George Washington University, Washington, DC, USA

E-mail: aalexan@gwu.edu

\section{Ivan Horváth}

University of Kentucky, Lexington, KY, USA

E-mail: horvath@pa.uky.edu

We study the chiral polarization properties of low-lying Dirac eigenmodes at finite temperature using the overlap operator. Results for pure gauge theory on both sides of deconfinement phase transition are presented. We find that the polarization scale decreases as we increase the temperature, but it remains non-zero as we cross in the deconfined phase and vanishes only when $T \approx 1.4 T_{c}$. This is caused by the presence of near-zero modes which, we find, are chirally polarized.

The 30th International Symposium on Lattice Field Theory

June 24 - 29, 2012

Cairns, Australia

\footnotetext{
${ }^{*}$ Speaker.
} 


\section{Introduction}

Banks-Casher relation connects the low-lying spectrum of the Dirac operator to the spontaneous symmetry breaking in QCD [1]. This relationship is rather generic and a more detailed understanding of the mechanism responsible for the chiral symmetry breaking is thought to be encoded in the chiral properties of the low lying eigenmodes. If we separate the chiral components of the Dirac eigenmode $D \psi=\lambda \psi$,

$$
\psi_{R}=\frac{1}{2}\left(1+\gamma_{5}\right) \psi \quad \text { and } \quad \psi_{L}=\frac{1}{2}\left(1-\gamma_{5}\right) \psi
$$

the relative magnitudes of these components at every lattice point, i.e., $\left\|\psi_{R, L}(x)\right\|$, carry information about the local chirality of the mode. For the eigenmodes of the free Dirac operator we have $\left\langle\psi\left|\gamma_{5}\right| \psi\right\rangle=0$ and, using translational symmetry for the chiral components magnitude, we can show that $\left\|\psi_{R}(x)\right\|=\left\|\psi_{L}(x)\right\|$. We say that these modes are anti-polarized since their left and right components have equal magnitude at every point.

In the presence of a gauge background, the chiral components of the eigenvector satisfy the following equations

$$
\begin{aligned}
& {\left[-D^{2}+\frac{1}{2} \sigma_{\mu \nu} F_{\mu v}^{S}\right] \psi_{L}=\lambda^{2} \psi_{L}, \quad F^{S}=\frac{1}{2}(F+\tilde{F}),} \\
& {\left[-D^{2}+\frac{1}{2} \sigma_{\mu v} F_{\mu v}^{A}\right] \psi_{R}=\lambda^{2} \psi_{R}, \quad F^{A}=\frac{1}{2}(F-\tilde{F}),}
\end{aligned}
$$

where $\tilde{F}_{\mu v}=\frac{1}{2} \varepsilon_{\mu v \alpha \beta} F_{\alpha \beta}$ is the dual gauge field tensor. Note that the equations above are similar to Schrödinger equations in four dimensions with $F^{S, A}$ playing the role of potential energy. For classical solutions of the gauge field equations, the gauge field itself is polarized, i.e., the field is either self-dual $\left(F^{A}=0\right)$ or anti-self-dual $\left(F^{S}=0\right)$ [2]. If the semi-classical approximation were relevant for QCD, one would expected that this tendency for polarization will not be destroyed by quantum fluctuations and there would be regions of the field where $F^{A}$ is strong and $F^{S}$ is weak and other regions where the situation is reversed. The equations above then imply that this tendency would also be reflected in the local chirality of the eigenmodes [3].

In a series of papers [3-6], a method to measure the effect of QCD dynamics on local chirality was proposed. Using this dynamical polarization, we found that for low-lying eigenmodes of the Dirac operator there is a small tendency for polarization, which turns into an anti-polarization tendency as we increase the magnitude of the eigenvalues $[5,6]$. The scale where the polarization turns into anti-polarization is the chiral polarization scale. We computed this scale on a series of quenched ensembles and showed that it survives in the continuum limit [5, 6]. The weak polarization of the low-lying modes is similar to the polarization observed for the dual components of the gauge field [7, 8].

The studies mentioned above were carried out for QCD at zero temperature where it is well known that the chiral symmetry is broken. If the polarization of the low-lying eigenmodes is indeed related to chiral symmetry breaking, we expect it to vanish when the symmetry is restored. In this study we compute the chiral polarization scale as we increase the temperature going from the chirally broken phase at low temperature to the chirally symmetric phase at high temperatures. 

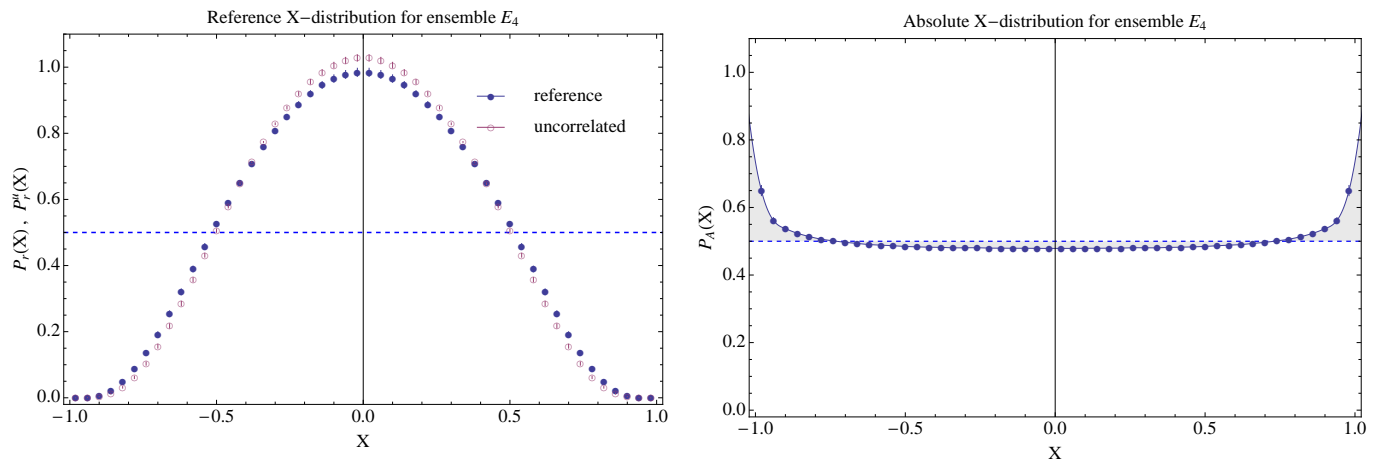

Figure 1: Left panel: The distribution of polarization variable in $\mathscr{P}$ (reference) and $\mathscr{P}^{u}$ (uncorrelated) for the lowest eigenmodes in ensemble $E_{4}$ [5]. Right panel: The absolute X-distribution for the same eigenmodes.

The plan of the paper is the following. In Section 2 we briefly review the tools used in this investigation: dynamical polarization and chiral polarization scale. In Section 3 we discuss the ensembles used in this study and show our results. Finally, in Section 4 we present our conclusions.

\section{Dynamical polarization and chiral polarization scale}

Dynamical polarization can be defined in a general context [5], but we will discuss it here in connection with local chirality. For a given set of eigenmodes of the Dirac operator evaluated on an ensemble of gauge configurations, each lattice point produces a pair of chiral components $q_{1,2}=$ $\left\|\psi_{R, L}(x)\right\|$. The probability distribution for these pairs is denoted with $\mathscr{P}\left(q_{1}, q_{2}\right)$. To measure the polarization of a given pair, one can use polarization variable $X=X\left(q_{1}, q_{2}\right)$, for example

$$
X=\frac{4}{\pi} \arctan \frac{q_{2}}{q_{1}}-1,
$$

the polar angle in $\left(q_{1}, q_{2}\right)$ plane rescaled to the interval $[-1,1]$. The probability distribution of $X$ in $\mathscr{P}$ assesses the degree of local chirality. However, this approach is kinematical since the shape of the final histogram is determined by the choice of polarization variable $X$.

To gauge the polarization induced by QCD dynamics, we compare $\mathscr{P}$ with a similar distribution, $\mathscr{P}^{u}$, where the correlation between the components is removed. The uncorrelated distribution $\mathscr{P}^{u}\left(q_{1}, q_{2}\right)=P_{1}\left(q_{1}\right) P_{2}\left(q_{2}\right)$ is generated using the marginal distributions $P_{1}\left(q_{1}\right)=\int d q_{2} \mathscr{P}\left(q_{1}, q_{2}\right)$ and $P_{2}\left(q_{2}\right)=\int d q_{1} \mathscr{P}\left(q_{1}, q_{2}\right)$. Note that due to symmetries of QCD we have $P_{1}=P_{2}$. For example, in the left panel of Fig. 1 we plot the histogram of one polarization variable $X$ for both correlated and uncorrelated distributions. Note that the correlated distribution is very similar to the uncorrelated one, but it is higher towards the extremal points and depressed in the middle. This is exactly what we expect from a polarized distribution. To better gauge this tendency, we define the absolute $X$-distribution using the polarization variable in which the uncorrelated distribution is constant. This distribution will be peaked towards the edges for polarized case; for anti-polarized case the absolute X-distribution will peak towards the middle. In the right panel of Fig. 1 we plot the absolute X-distribution for the same ensemble. We see that this indeed confirms that the correlated distribution is polarized. 

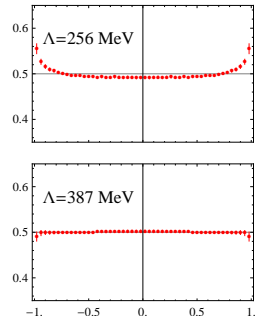
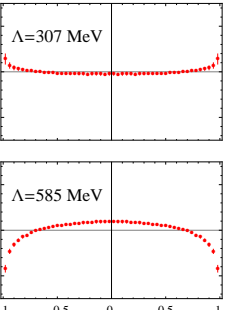
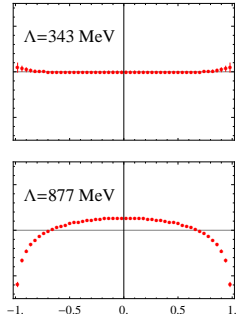

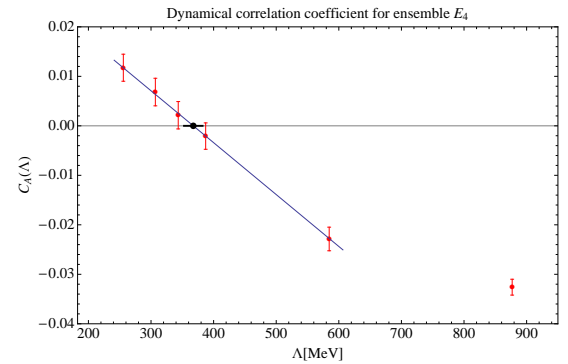

Figure 2: Left panel: Absolute X-distribution as a function of scale for the same ensemble as in Fig. 1. Right panel: Correlation coefficient as a function of scale.

As we noted in the introduction, the low-lying modes of the Dirac operator in QCD at zero temperature are weakly polarized [5]. In the left panel of Fig. 2 we plot the absolute X-distribution for eigenmodes at different scales. As we increase the eigenvalue this tendency weakens and somewhere in the interval $343 \mathrm{MeV} \leq \Lambda \leq 387 \mathrm{MeV}$ the polarization vanishes. To define the chiral polarization scale where the polarization vanishes, we use the correlation coefficient of chiral polarization [5]

$$
C_{A}=2 \Gamma-1 \quad \text { with } \quad \Gamma=\int_{-1}^{1} d X|X| P_{A}(X),
$$

where $P_{A}(X)$ is the absolute $\mathrm{X}$-distribution. Note that $\Gamma$ is the probability that a pair drawn from $\mathscr{P}$ is more polarized than another independently drawn from $\mathscr{P}^{u}$. If $\mathscr{P}$ is the same as $\mathscr{P} u$, we have no polarization and $\Gamma=1 / 2$. The correlation coefficient is normalized to be 0 in this situation, positive for polarized distributions and negative for anti-polarized ones. In the right panel of Fig. 2 we plot the correlation coefficient, which allows us to easily extract the chiral polarization scale for the ensemble, $\Lambda_{T}=368(15) \mathrm{MeV}$.

\section{Results}

To explore the connection between eigenmodes' polarization and chiral symmetry breaking, we measured the chiral polarization scale at different temperatures, both below and above the deconfinement transition. We generated a set of quenched ensembles using Wilson gauge action with $\beta=6.054$. This value of $\beta$ corresponds to a lattice spacing of $a / r_{0}=0.170$ according to a non-perturbative parametrization [9]. Using $r_{0}=0.5 \mathrm{fm}$ we get $a=0.085 \mathrm{fm}$. To avoid issues related to changing the cutoff, we varied the temperature by changing the temporal extent while keeping the lattice spacing fixed. The spatial volume was kept fixed at $V=(20 a)^{3}=(1.7 \mathrm{fm})^{3}$. The parameters for our ensembles are presented in Table 1.

\begin{tabular}{l|cccccccc}
$N_{t}$ & 4 & 6 & 7 & 8 & 9 & 10 & 12 & 20 \\
\hline$N_{\text {conf }}$ & 100 & 100 & 400 & 400 & 200 & 200 & 200 & 100 \\
\hline$T / T_{c}$ & 2.09 & 1.39 & 1.20 & 1.05 & 0.93 & 0.84 & 0.70 & 0.42
\end{tabular}

Table 1: Parameters for the ensembles used in this study. The temperature is determined using the lattice spacing and the critical temperature $T_{c}=277 \mathrm{MeV}[10]$. 

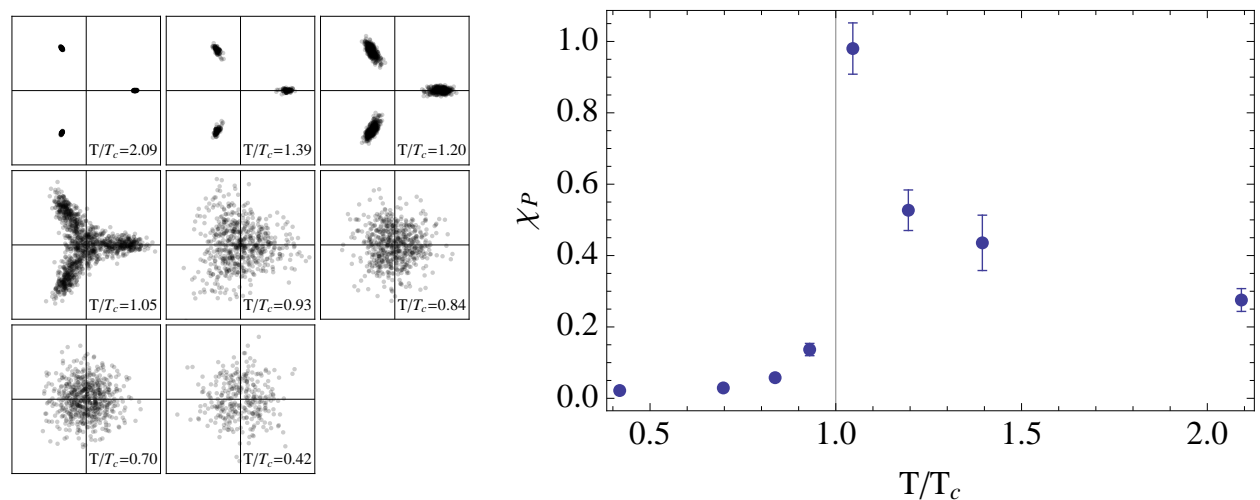

Figure 3: Polyakov loop distribution (left) and susceptibility (right) for our ensembles.

Since there is an uncertainty in scale setting of the order of $5 \%$, the temperature is determined in units of string tension [10] and the lattice spacing in units of $r_{0}$ [9], we decided to compute the Polyakov loop susceptibility to confirm that the transition temperature is determined correctly. In Fig. 3 we plot the distribution of the Polyakov loop on our ensembles in the left panel and the susceptibility in the right panel. From these figures is apparent that we have the temperature scale determined correctly.

Pure glue theory has a $Z(3)$ symmetry that is reflected in the distribution of the Polyakov loop, as can be seen from the left panel of Fig. 3. In the deconfined phase this symmetry is spontaneously broken and the spectrum of the Dirac operator is very different for configuration from different $Z(3)$ sectors [11]. Dynamical quarks break this symmetry explicitly and bias the theory towards configurations with Polyakov loop in the real sector $(-\pi / 3 \leq \arg P \leq \pi / 3)$. Since for the full theory only the real sector is relevant, in this study we only use configurations in this sector.

To compute the chiral polarization scale, we bin the eigenvalues in bins of width $\delta \lambda=50 \mathrm{MeV}$ and compute the average $C_{A}(\lambda)$ for each bin. In the left panel of Fig. 4 we plot the average $C_{A}$ as a function of $\lambda$ for each of ensemble where $C_{A}(\lambda=0)>0$; these are the ensemble with $T / T_{c} \leq 1.20$ where the low-lying modes are polarized. Note that, as in the zero temperature case, at a sufficiently large $\lambda$ the modes become anti-polarized. The chiral polarization scale is computed for each ensemble using a simple linear fit for the bins that bracket the transition scale. The error bars are determined using the jackknife method. The results are presented in the right panel of Fig. 4. As expected, the chiral polarization scale decreases as we increase the temperature. A bit surprising is the fact that it does not vanish at $T=T_{c}$. Before we discuss the cause for this phenomenon, we note that if we fit to a simple ansatz, $\Lambda(T)=\alpha\left(T-T_{c}\right)^{\beta}$, using $T_{c}, \alpha$ and $\beta$ as free parameters, we find that the value of $T_{c}$ extracted from this fit is very close to the real value. In this fit we only use the data points with $T<T_{c}$.

The reason chiral polarization scale does not vanish as we cross the phase transition is connected to the fact that the spectral density at the edge of the spectrum, $\rho(\lambda=0)$, does not vanish immediately above $T_{c}$. These near-zero modes are polarized and lead to a non-vanishing chiral polarization scale. To show this, in Fig. 5 we plot the eigenvalue and correlation coefficient for each mode in our ensembles. Note that at low temperature $\rho(0)>0$ as required by Banks-Casher 


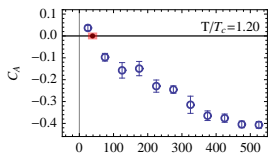

$\lambda[\mathrm{MeV}]$
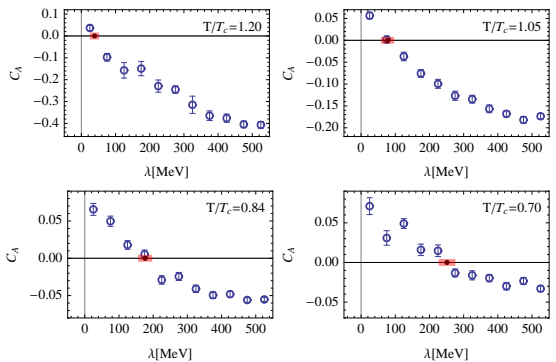

$\lambda[\mathrm{MeV}]$
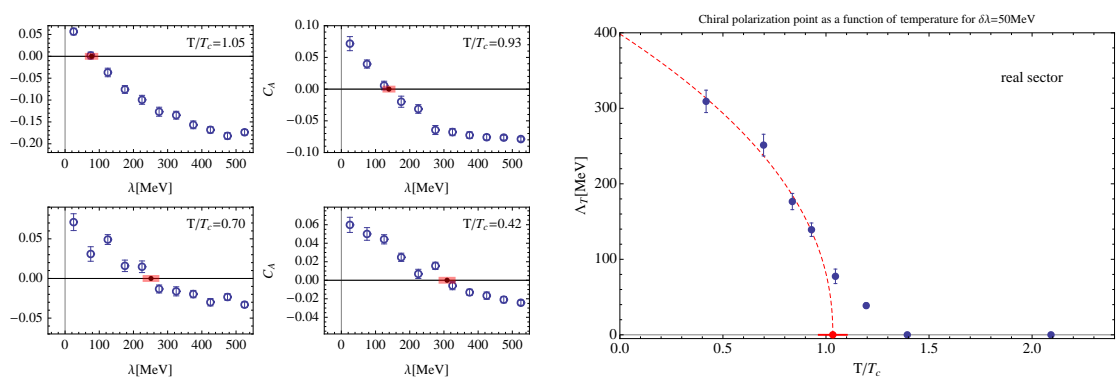

Figure 4: Left panel: Correlation coefficient as a function of eigenmode scale. Right panel: Chiral polarization scale as a function of temperature. The dashed line indicate the fit discussed in the text.

relation in a phase where chiral symmetry is spontaneously broken; these near-zero modes are mostly polarized $\left(C_{A}>0\right)$. The standard expectation is that above $T_{c}$ the Dirac spectrum develops a gap and the symmetry is restored. This is indeed consistent with what we observe for $T>1.4 T_{c}$ and the modes at the low end of the spectrum are strongly anti-polarized. However, at intermediate temperatures, $T_{c}<T<1.4 T_{c}$, the situation is more complex: as remarked earlier, a small density of near zero modes remains and these modes are polarized. The existence of near-zero modes above deconfinement transition was observed earlier [12]. We emphasize here that these are not zero modes, which are easy to distinguish when using overlap operator. We removed the zero-modes from our analysis.

\section{Conclusions}

We computed the chiral transition scale as a function of temperature for pure gauge configurations. We find that the scale decreases as we approach the phase transition. If we extrapolate from the confined phase, the polarization scale seems to vanish very near $T_{c}$. However, direct calculations show that it vanishes for $T \approx 1.4 T_{c}$. The discrepancy is due to the presence of nearzero modes at temperatures as high as $1.2 T_{c}$. These modes are polarized causing the polarization scale to be non-zero. This suggests that the deconfinment temperature and the chiral restoration scale - defined via the condition that $\rho(0)=0$ - differ in the pure gauge theory.

The near zero modes, which are connected to chiral symmetry breaking, are almost all (weakly) polarized. A more precise framework for discussing the polarization properties of the Dirac eigenmodes and their connection to chiral symmetry breaking is presented in [13].

Acknowledgments: The computational resources for this project were provided in part by the George Washington University IMPACT initiative. This work is supported in part by the DOE grant DE-FG02-95ER-40907 and NSF CAREER grant PHY-1151648.

\section{References}

[1] T. Banks and A. Casher, Chiral Symmetry Breaking in Confining Theories, Nucl.Phys. B169 (1980) 103.

[2] T. Schafer and E. V. Shuryak, Instantons in QCD, Rev.Mod.Phys. 70 (1998) 323-426, [hep-ph/9610451]. 

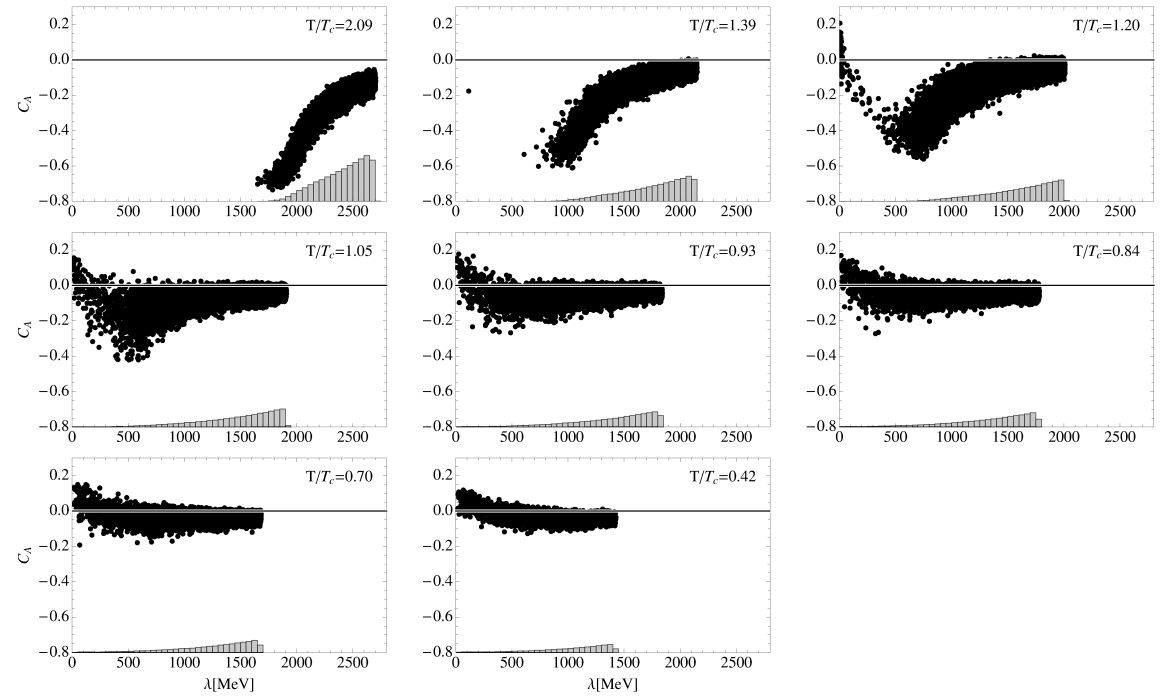

Figure 5: Correlation coefficient versus eigenmode scale for all ensembles in this study. The band below indicates the spectral density $\rho(\lambda)$.

[3] I. Horváth, N. Isgur, J. McCune, and H. B. Thacker, Evidence against instanton dominance of topological charge fluctuations in QCD, Phys. Rev. D65 (2002) 014502, [hep-lat/ 0102003 ].

[4] T. Draper, A. Alexandru, Y. Chen, S.-J. Dong, I. Horváth, et. al., Improved measure of local chirality, Nucl.Phys.Proc.Suppl. 140 (2005) 623-625, [hep-lat/ 040800 6].

[5] A. Alexandru, T. Draper, I. Horváth, and T. Streuer, The Analysis of Space-Time Structure in QCD Vacuum II: Dynamics of Polarization and Absolute X-Distribution, Annals of Physics 326 (2011) 1941-1971, [arXiv:1009.4451].

[6] A. Alexandru, T. Draper, I. Horváth, and T. Streuer, Absolute Measure of Local Chirality and the Chiral Polarization Scale of the QCD Vacuum, PoS LATTICE2010 (2010) 082, [arXiv:1010.5474].

[7] A. Alexandru and I. Horváth, How Self-Dual is QCD?, Phys.Lett. B706 (2012) 436-441, [arXiv:1110.2762].

[8] A. Alexandru and I. Horváth, Absolute X-distribution and self-duality, PoS LATTICE2011 (2011) 268, [arXiv:1111.3897].

[9] ALPHA Collaboration, M. Guagnelli, R. Sommer, and H. Wittig, Precision computation of a low-energy reference scale in quenched lattice QCD, Nucl. Phys. B535 (1998) 389-402, [hep-lat/9806005].

[10] F. Karsch and E. Laermann, Thermodynamics and in medium hadron properties from lattice QCD, hep-lat/0305025. Prepared for Quark-Gluon Plasma III, R. Hwa (ed.).

[11] S. Chandrasekharan and N. H. Christ, Dirac spectrum, axial anomaly and the QCD chiral phase transition, Nucl.Phys.Proc.Suppl. 47 (1996) 527-534, [hep-lat/950 9095].

[12] R. G. Edwards, U. M. Heller, J. E. Kiskis, and R. Narayanan, Chiral condensate in the deconfined phase of quenched gauge theories, Phys.Rev. D61 (2000) 074504, [hep-lat/9910041].

[13] A. Alexandru and I. Horváth, Spontaneous Chiral Symmetry Breaking as Condensation of Dynamical Chirality, arXiv:1210.7849. 